\title{
An Atomistic-based Spring-mass Finite Element Approach for Vibration Analysis of Carbon Nanotube Mass Detectors
}

\author{
S.K. Georgantzinos and N.K. Anifantis \\ Machine Design Laboratory \\ Mechanical and Aeronautics Engineering Department \\ University of Patras, GR 26500
}

Greece

\section{Introduction}

Since their discovery in 1991 by Ijima [1], carbon nanotubes (CNT) have received much attention as a new class of nanomaterials revealing a significant potential for use in a diverse range of novel and evolving applications. Much of the interest in CNTs has focused on their particular molecular structures and their unique electronic and mechanical properties. For example, their elastic stiffness is comparable to that of diamond (1000 GPa), while their strength is ten times larger (yield strength $100 \mathrm{GPa}$ ). Furthermore, CNTs conduct heat and electricity along their length with very little resistance, and therefore they act as tiny electrical wires or paths for the rapid diffusion of heat. As a result, progressive research activities regarding CNTs have been ongoing in recent years. For more detail on theoretical predictions and experimental measurements of both mechanical and physical properties, see the comprehensive reviews in $[2,3]$.

The combination of an extremely high stiffness and light weight in CNTs results in vibration frequencies on the order of $\mathrm{GHz}$ and $\mathrm{THz}$. There is a wide range of applications in which the vibrational characteristics of CNTs are significant. In applications such as oscillators, charge detectors, field emission devices, vibration sensors, and electromechanical resonators, oscillation frequencies are key properties. An representative application is the development of sensors for gaseous molecules, which play significant roles in environmental monitoring, chemical process control, and biomedical applications. Mechanical resonators are widely used as inertial balances to detect small quantities of adsorbed mass through shifts in oscillation frequency. Recently, advances in lithography and materials synthesis have enabled the fabrication of nanoscale mechanical resonators that utilize CNTs $[4,5]$. The use of a CNT to make the lightest inertial balance ever is essentially a target to make a nanoscale mass spectrometer of ultrahigh resolution. Building such a mass spectrometer that is able to make measurements with atomic mass sensitivity is one of the main goals in the burgeoning field of nanomechanics. An inertial balance relies only on the mass and does not, therefore, require the ionization or acceleration stages that might damage the molecules being 
measured. This means that a nanoscale inertial balance would be able to measure the mass of macromolecules that might be too fragile to be measured by conventional instruments [5]. Several efforts for the building of CNT-based sensors have been presented in the literature. Mateiu et al. [6] described an approach for building a mass sensor based on multi-walled CNTs with an atomic force microscope. Chiu et al. [7] demonstrated atomic-scale mass sensing using doubly clamped, suspended CNT resonators in which their single-electron transistor properties allowed the self-detection of nanotube vibration. They used the detection of shifts in the resonance frequency of the nanotubes to sense and determine the inertial mass of atoms as well as the mass of the nanotube itself. Commonly, multi-walled CNTs are less sensitive than single-walled CNTs. However, multi-walled carbon nanotubes are easier to manipulate and more economical to be produced, since they are both longer and have larger diameters than single-walled CNTs [8].

Hence, it is important to develop accurate theoretical models for evaluation of natural frequencies and mode shapes of CNTs. An excellent review article was recently published by Gibson et al. [9] that presents related scientific efforts in dealing with the vibrational behavior of CNTs and their composites, including both theoretical and experimental studies. Controlled experiments performed at nanoscale dimensions remain both difficult and expensive. Despite of this fact, Garcia-Sanchez et al. [10] have recently presented a mechanical method for detecting CNT resonator vibrations using a novel scanning force microscopy method. The comparison between experimental and theoretical methods prerequire the complete definition of all parameters such as the length of the vibrating nanotube, the nanotube type and other conditions that influence the vibrational behavior such as the slack phenomenon, nature of the support condition, environmental conditions and other influences.

In an attempt to approach the vibration behavior of CNTs, various theoretical methods have been reported in literature. Molecular dynamics (MD) and molecular mechanics, as well as elastic continuum mechanics, are considered efficient because they can accurately and costeffectively produce results that closely approximate the behavior of CNTs. . Each of the previously mentioned approaches offers different advantages, but also certain drawbacks. MD is an accurate method capable of simulating the full mechanical CNT performance. However, it carries a high computational cost that may be prohibitive for large-scale problems, especially in the context of vibration analysis. Molecular mechanics-based techniques, such as those in [11-13], have been used for vibration analysis of CNTs and have been shown to be accurate and also more computationally cost-effective than MD. Nevertheless, under such approaches, the modeling of atomic interactions requires special attention because the mechanical equivalent used to simulate the carbon-carbon bond deformations must be efficient for the studied problem. Generally, typical elements of classical mechanics, such as rods [14], beams [15, 16], springs [17-19] and cells [20] have been proposed including appropriate stiffness parameters, thus their strain energies are equivalent to the potential energies of each interatomic interaction. Furthemore, elastic continuum mechanics methods based on well-known beam theories have also been successfully used to evaluate the vibration characteristics of CNTs under typical boundary conditions [21-24]. Xu et al. [25] studied the free vibration of double-walled CNTs modeled as two individual beams interacting with each other taking van der Waals forces into account and supported with different boundary conditions between the inner and outer tubes. These methods have the lowest computational cost; however, they can compute only a subset (mainly the bending modes) of the vibrational modes and natural frequencies. 
In terms of CNT mass detector function, the principle of mass detection using CNT-based resonators is based on the fact that the vibrational behavior of the resonator is sensitive to changes in its mass due to attached particles. The change of the resonator mass due to an added mass causes frequency shifts. The key challenge in mass detection is quantifying the changes in the resonant frequencies due to added masses. Based on this principle, the usage of computational tools, as presented in prevous paragraph, capable of simulating the vibrational behavior of CNT-based mass detectors is important for two reasons. First, they can cost-effectively predict the mass sensing characteristics of different nanoresonator types, thereby allowing the optimal design of detectors with a specific sensing range. Second, their cooperation with experimental measurements can improve the detection abilities of the nanodevice. With respect to theoretical studies on CNT-based sensors, Li and Chou [26] examined the potential of nanobalances using individual single-walled CNTs in a cantilevered or bridged configuration. Wu et al. [27] explored the resonant frequency shift of a fixed-free single-walled CNT caused by the addition of a nanoscale particle to the beam tip. This was done to explore the suitability of a single-walled CNT as a mass detector device in a micro-scale situation via a continuum mechanics-based finite element method simulation using a beam-bending model. Chowdhury et al. [28] examined the potential of single-walled CNTs as biosensors using a continuum mechanics-based approach and derived a closed-form expression to calculate the mass of biological objects from the frequency shift.

In this chapter, an atomistic spring-mass based finite element approach appropriate to simulate the vibration characteristics of single-walled and multi-walled CNTs is presented. The method uses spring-mass finite elements that simulate the interatomic interactions and the inertia effects in CNTs. It uses a special technique for simulating the bending between adjacent bonds, distinguishing it from other mechanics-based methods. This method utilizes the exact microstructure of the CNTs while allowing the straightforward input of the force constants that molecular theory provides. In addition, spring-like elements are formulated in order to simulate the interlayer van der Waals interactions. These elements connect all atoms between different CNT layers at a distance smaller than the limit below which the van der Waals potential tends to zero. The related stiffness is a function of this distance. The resulting dynamic equilibrium equations can be used to generate new results. Results available in the literature were used to validate the proposed method. Parametric analyses are performed reporting the natural frequencies as well as the mode shapes of various multi-walled CNTs for different aspect geometric characteristics. Furthermore, the principle of mass detection using resonators is based on the fact that the resonant frequency is sensitive to the resonator mass, which includes the self-mass of the resonator and the attached mass. The change of the attached mass on the resonator causes a shift to the resonant frequency. Since, the key issue of mass detection is in quantifying the change in the resonant frequency due to the added mass, the effect of added mass to the vibration signature of CNTs is investigated for the clamped-free and clamped-clamped support conditions. And different design parameters. Additionally, the frequency shifts of singleand multi-walled CNTs were compared.

\section{CNTs geometry}

A planar layer of carbon atoms forms a periodic structure called the graphene sheet. Pencil lead consists of a stack of overlaying graphene sheets that easily separate upon shearing in 
writing. A perfect graphene sheet in the xy-plane consists of a doubly periodic hexagonal lattice defined by two base vectors,

$$
\mathbf{v}_{\mathbf{1}}=a(1,0) \text { and } \mathbf{v}_{\mathbf{2}}=a\left(\cos 60^{\circ}, \sin 60^{\circ}\right)=a\left(\frac{1}{2}, \frac{\sqrt{3}}{2}\right)
$$

where $\alpha$ is equal to $\sqrt{3} r_{h}$ and $r_{h}$ is the radius of the hexagonal cell. Note that the lengths of these vectors are equal. Any point of plane $P=(x, y)$ is uniquely defined as a linear combination of these two vectors,

$$
\mathbf{P}=\mathbf{v}_{0}+a \mathbf{v}_{1}+b \mathbf{v}_{2},
$$

where $a$ and $b$ are integers, provided that $\mathbf{v}_{0}$ is the center of a hexagon.

Knowing the geometry of graphene, a single-walled CNT can be geometrically generated by rolling a single-layer graphene sheet, which is ideally cut, to make a cylinder. The graphene sheet must be rolled up in the direction of the chiral vector $\mathbf{C}_{\mathbf{h}}$ defined as (see Figure 1):

$$
\mathbf{C}_{\mathbf{h}}=n \mathbf{a}_{1}+m \mathbf{a}_{2}
$$

where $\mathbf{a}_{1}$ and $\mathbf{a}_{2}$ are the basis vectors of the honeycomb lattice and integers $(n, m)$ are the number of steps along the zigzag carbon bonds and generally are used to name a nanotube.

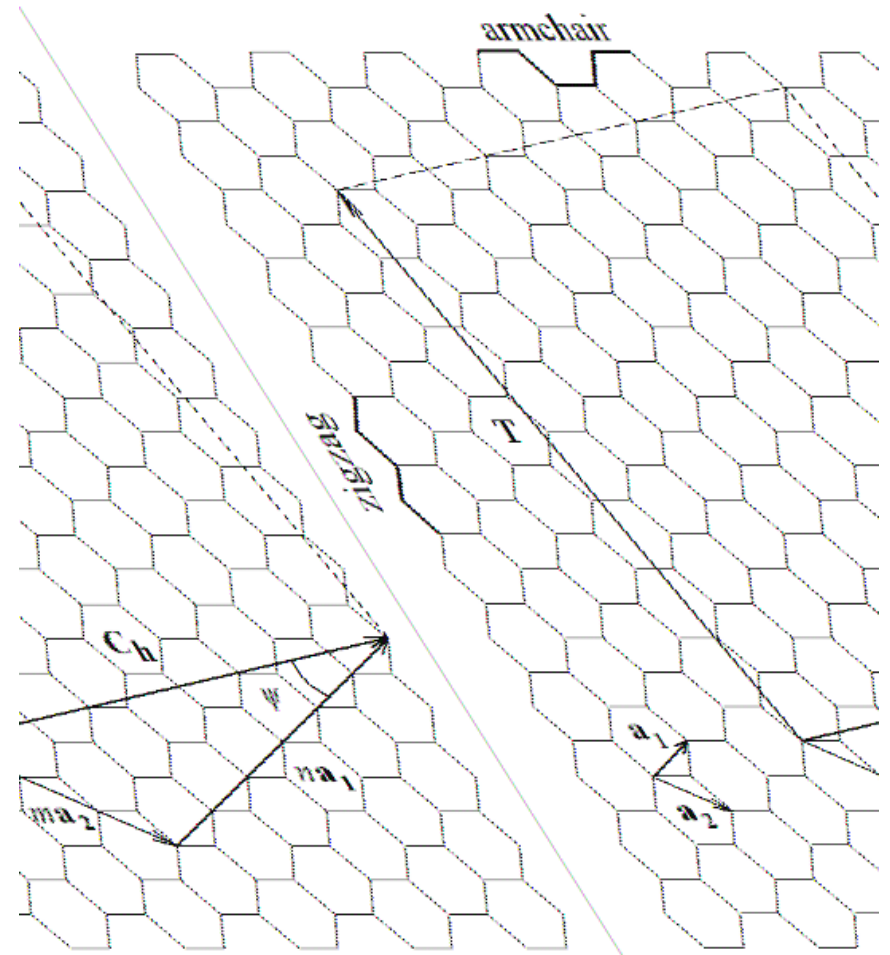

Fig. 1. Generation of a SWCNT from a graphene sheet. 
A nanotube $(n, n)$ is usually named as armchair (Figure $2(a))$ while the nanotube $(n, 0)$ is usually named zigzag (Figure 2(b)). The chiral angle $\psi\left(0 \leq \psi \leq 30^{\circ}\right)$ is defined as:

$$
\tan \psi=\frac{\sqrt{3} m}{(2 n+m)}
$$

It is obvious that for an armchair nanotube $\psi=30^{\circ}$ while for a zigzag $\psi=0^{\circ}$. The nanotube's diameter $D$ is given by the following equation:

$$
D=\frac{a_{\mathrm{c}-\mathrm{c}} \sqrt{3\left(n^{2}+n m+m^{2}\right)}}{\pi}
$$

where $a_{\mathrm{c}-\mathrm{c}}$ is distance between two neighbor carbon atoms and is equal to $0.1421 \mathrm{~nm}$.

Chiral vector $C_{h}$ and the following translational vector $T$ define the ideal rectangular cutting area of graphene sheet:

$$
\mathbf{T}=\left(\frac{2 m+n}{W}\right) \mathbf{a}_{1}+\left(-\frac{2 n+m}{W}\right) \mathbf{a}_{2}
$$

where $W$ defines the higher common divisor of $2 m+n$ and $2 n+m$.

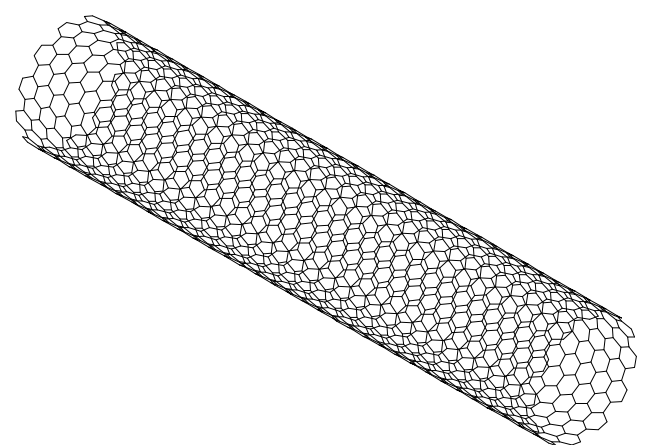

(a)

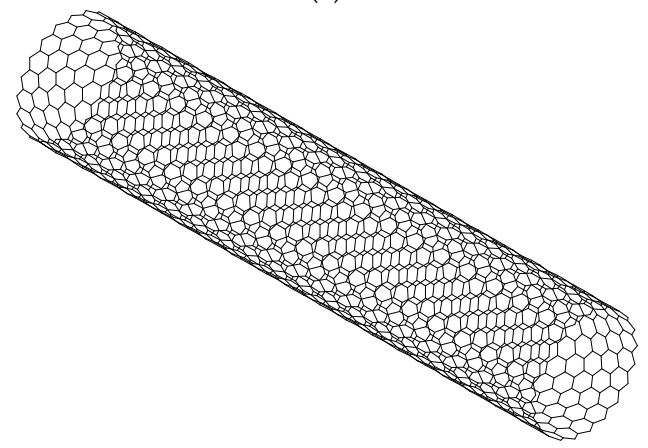

(b)

Fig. 2. Geometry of an (a) armchair and (b) zigzag single-walled CNTs 
For simplicity, the original coordinate system of the graphene sheet $\left(x^{\prime}, y^{\prime}\right)$ is transformed into a new system $(x, y, z)$ of the nanotube such that $\mathbf{T}$ is along $y^{\prime}$-axis. Then, the graphene atomic coordinates are converted to those of the nanotube according to the equation (Kołoczek et al. [29]):

$$
(x, y, z)=\left(R \cos \left(\frac{x^{\prime}}{R}\right), r \sin \left(\frac{x^{\prime}}{R}\right), y^{\prime}\right)
$$

where $R$ is the nanotube's radius.

A multi-walled CNT consists of multiple layers of graphene rolled in on themselves to form a tube shape. In other words, every multi-walled CNT consists of more than one coaxial single-walled CNTs. Since single-walled CNTs are parts of multi-walled CNTs, the layers of multi-walled CNTs have similar geometric characteristics. Given that the interlayer distance is $0.34 \mathrm{~nm}$, as has been observed in [1], the difference between diameters of neighbouring layers, where the diameter of every layer can be calculated using the Equation (5), is $\Delta D=0.68 \mathrm{~nm}$. Knowing that this equation is a function only of chirality indexes of the two neighboring nanotubes in a multi-walled CNT, someone can calculate the convenient types of single-walled CNTs able to apart the multi-walled CNT of

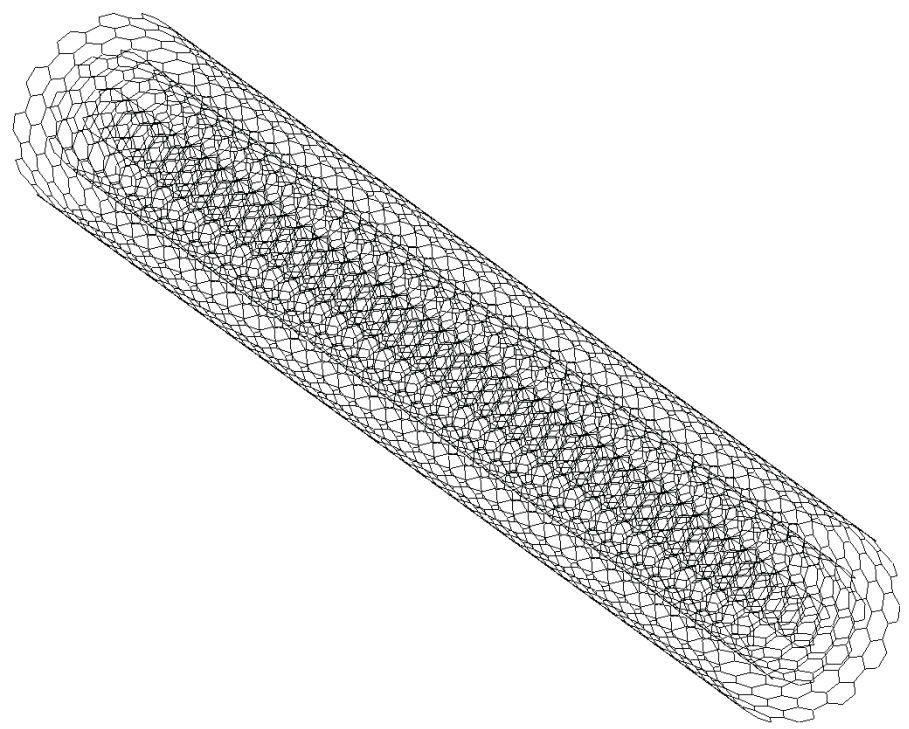

Fig. 3. Geometry of a multi-walled CNT.

specific number of layers and outer diameter. If $\left(n_{1}, m_{1}\right)$ and $\left(n_{2}, m_{2}\right)$ are the types of the inner and outer neighboring layers respectively, it is observed that for zig-zag nanotubes, the chirality indexes are $n_{2}=n_{1}+9$ and $m_{2}=m_{1}=0$. Correspondingly, if the neighboring nanotubes are armchair then $n_{2}=m_{2}=n_{1}+5$ and $m_{1}=n_{1}$. The type of one MWCNT, here, is declared as the sequence of the types of all layers $\left(n_{\text {in }}, m_{\text {in }}\right)-\ldots-\left(n_{\text {out }}, m_{\text {out }}\right)$, starting from the type of the innermost tube and finishing to the type of the outermost tube. A representative example of multi-walled CNT geometry consists of three layers is depicted in Figure 3. 


\section{Computational model}

\subsection{Force field}

The total potential energy, omitting non-bonded interactions, i.e. the electrostatic energy and the energy due to van der Waals interaction, is a sum of energies caused by the bonded interatomic interactions, which are depicted in Figure 4(a), and may be expressed by the following equation (Rappe et al. [30]):

$$
U=\sum U_{r}+\sum U_{\theta}+\sum U_{\phi}+\sum U_{\omega}
$$

where $U_{r}$ represents the energy due to bond stretching, $U_{\theta}$ the energy due to bond angle bending, $U_{\phi}$ the energy due to dihedral angle torsion and $U_{\omega}$ the energy due to out of plane torsion.

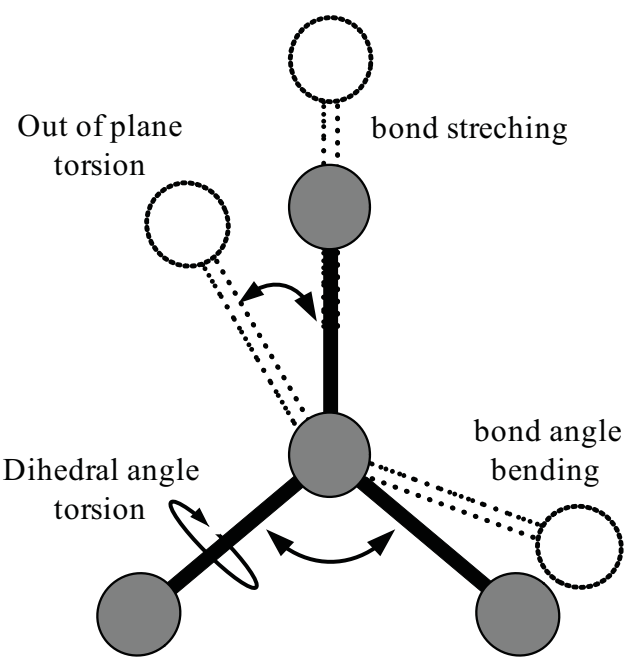

(a)

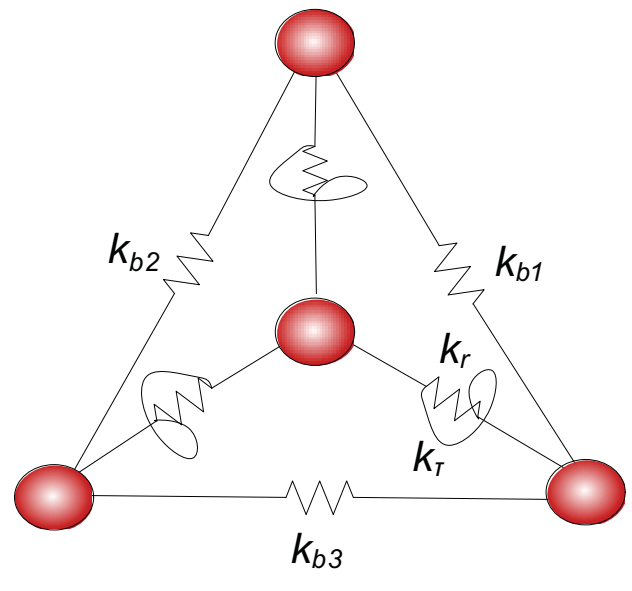

(b)

Fig. 4. Force field in neighboring atoms (a) interatomic interactions and (b) spring model.

Under the assumption of small deformations, the harmonic approximation is adequate for describing potential energy (Gelin [31]) and therefore the force field. By adopting the simplest harmonic forms and combining the dihedral angle, torsion with the out of plane torsion into a single equivalent term then the following terms can describe the total potential energy [17]:

$$
\begin{gathered}
U_{r}=\frac{1}{2} k_{r}(\Delta r)^{2}, \quad \frac{d^{2} U_{r}}{d \Delta r^{2}}=k_{r} \\
U_{\theta}=\frac{1}{2} k_{\theta}(\Delta \theta)^{2}, \quad \frac{d^{2} U_{\theta}}{d \Delta \theta^{2}}=k_{\theta} \\
U_{\tau}=U_{\varphi}+U_{\omega}=\frac{1}{2} k_{\tau}(\Delta \phi)^{2}, \quad \frac{d^{2} U_{\tau}}{d \Delta \tau^{2}}=k_{\tau}
\end{gathered}
$$


where $k_{r}, k_{\theta}$ and $k_{\tau}$ are the bond stretching, bond angle bending, and torsional resistance force constants, respectively, while $\Delta r, \Delta \theta$ and $\Delta \phi$ represent the bond length, bond angle and twisting bond angle variations, respectively.

The second derivatives of the potential energy terms appearing in equations (7), (8) and (9) with respect to bond stretch, bond angle and twisting bond angle variations, respectively, produce spring stiffness coefficients $k_{r}, k_{\theta}$ and $k_{\tau}$. Thus, here, axial and torsional springs that straightforwardly introduce the physical constants are utilized (Figure 4(b)) in order to describe the force field. The springs interconnect the nodes that have been extracted from the eq. (3). The bond angle bending interaction is simulated by axial springs, which have stiffness

$$
k_{b i}=\left(\frac{1}{a_{\mathrm{c}-\mathrm{c}} \cos \left(90^{\circ}-\gamma\right)}\right)^{2} k_{\theta}
$$

as has been described in [17], where $\gamma=30^{\circ}$ in the hexagonal lattice of the graphene. This angle may vary for each $\mathrm{C}-\mathrm{C}-\mathrm{C}$ microstructure in a CNT according to its type and radius due to its cylindrical shape. In the case of chiral nanotubes, the stiffness of the three different bending springs (Figure $4(\mathrm{~b})$ ) varies $k_{b 1} \neq k_{b 2} \neq k_{b 3}$. In the cases of armchair and zigzag nanotubes, two of the three bending spring stiffnesses are equal due to the same angle $\gamma$. In the other hand, because of the planar shape of the graphene sheets, all the bond angle bending springs have the same stiffness, i.e. $k_{b 1}=k_{b 2}=k_{b 3}$.

The interlayer interactions between the walls of a multi-walled CNT is caused by the van der Waals forces and can described through the Lennard-Jones pair potential $[32,33]$

$$
U(R)=4 \varepsilon\left[\left(\frac{\sigma}{R}\right)^{12}-\left(\frac{\sigma}{R}\right)^{6}\right]
$$

where $\mathrm{R}$ is the distance between the interacting atoms, $\varepsilon$ is the depth of the potential and $\sigma$ is a parameter that is determined by the equilibrium distance. The van der Waals force $F$ is obtained by taking the derivative of the Lennard-Jones pair potential, i.e.,

$$
F(R)=-\frac{\mathrm{d} U(R)}{\mathrm{d} r}=-4 \varepsilon\left(6 \frac{\sigma^{6}}{R^{7}}-12 \frac{\sigma^{12}}{R^{13}}\right)
$$

It should be noted that the initial pressure exerted on a sheet is negligible at the equilibrium distance, and thus the van der Waals force can be estimated by the Taylor expansion to the first order around the equilibrium position, i.e.,

$$
F(R)=F\left(R_{0}\right)+\frac{\mathrm{d} F\left(R_{0}\right)}{\mathrm{d} R}\left(R-R_{0}\right)=-24 \varepsilon\left(26 \frac{\sigma^{12}}{R_{0}^{13}}-7 \frac{\sigma^{6}}{R_{0}^{7}}\right)\left(R-R_{0}\right)
$$

Where $R_{0}=\sqrt{\left(X_{j}-X_{i}\right)^{2}+\left(Y_{j}-Y_{i}\right)^{2}+\left(Z_{j}-Z_{i}\right)^{2}}$ is the initial distance between the atoms of the different layers. 


\subsection{CNT modeling}

In order to evaluate the vibrational characteristics of CNTs, we must develop equations that describe the dynamic equilibrium of the entire model. The elemental equations must be constructed first before the global stiffness and mass matrices can be assembled.

The elemental equation for the $a_{i}$-element, as defined and developed in [18] to represent the bond stretching as well as twisting bond angle interactions, is

where

$$
\left[\begin{array}{cc}
k_{r} \mathbf{I}_{\mathbf{2 0}} & \mathbf{0} \\
\mathbf{0} & k_{\tau} \mathbf{I}_{\mathbf{2 0}}
\end{array}\right]\left[\begin{array}{l}
\mathbf{u} \\
\boldsymbol{\theta}
\end{array}\right]+\left[\begin{array}{cc}
m_{r} \mathbf{I}_{2 \mathbf{1}} & \mathbf{0} \\
\mathbf{0} & \mathbf{0}
\end{array}\right]\left[\begin{array}{l}
\ddot{\mathbf{u}} \\
\ddot{\boldsymbol{\theta}}
\end{array}\right]=\left[\begin{array}{c}
\mathbf{F} \\
\mathbf{T}
\end{array}\right] \text { or } \quad \mathbf{k}_{\mathbf{a}} \mathbf{U}+\mathbf{m}_{\mathbf{a}} \ddot{\mathbf{U}}=\mathbf{P},
$$

$$
\mathbf{I}_{20}=\left[\begin{array}{cc}
1 & -1 \\
-1 & 1
\end{array}\right], \mathbf{I}_{21}=\left[\begin{array}{cc}
0 & -1 \\
0 & 1
\end{array}\right],
$$

$m_{r}$ is the concentrated mass equal to the half or whole mass of the carbon nuclei [18], $\mathbf{F}$ represents the forces applied to nodes 1 and 2 of the element, $\mathbf{u}$ is the vector of nodal displacements $\left(\mathbf{u}=\left[\begin{array}{ll}u_{1} & u_{2}\end{array}\right]^{\mathrm{T}}, \mathbf{F}=\left[\begin{array}{ll}F_{1} & F_{2}\end{array}\right]^{\mathrm{T}}\right), \boldsymbol{\theta}$ is the vector of nodal rotations, $\mathbf{T}$ is the vector of the applied torsional moments $\left(\boldsymbol{\theta}=\left[\begin{array}{ll}\theta_{1} & \theta_{2}\end{array}\right]^{\mathrm{T}}, \mathbf{T}=\left[\begin{array}{ll}T_{1} & T_{2}\end{array}\right]^{\mathrm{T}}\right), \mathbf{U}$ is the vector of nodal translations and rotations, $\ddot{\mathbf{U}}$ is the vector of nodal accelerations, $\mathbf{P}$ is the column vector of loads, and finally, $\mathbf{k}_{\mathbf{a}}$ and $\mathbf{m}_{\mathbf{a}}$ are the elemental stiffness and mass matrix, respectively. Similarly, the equation for the $b_{i}$-element, which describes the bond angle interaction in the hexagonal lattice, is

$$
\left[\begin{array}{cc}
k_{b} \mathbf{I}_{20} & \mathbf{0} \\
\mathbf{0} & \mathbf{0}
\end{array}\right]\left[\begin{array}{l}
\mathbf{u} \\
\boldsymbol{\theta}
\end{array}\right]+\left[\begin{array}{ll}
\mathbf{0} & \mathbf{0} \\
\mathbf{0} & \mathbf{0}
\end{array}\right]\left[\begin{array}{c}
\ddot{\mathbf{u}} \\
\ddot{\boldsymbol{\theta}}
\end{array}\right]=\left[\begin{array}{c}
\mathbf{F} \\
\mathbf{T}
\end{array}\right] \text { or } \mathbf{k}_{\mathbf{b}} \mathbf{U}+\mathbf{m}_{\mathbf{b}} \ddot{\mathbf{U}}=\mathbf{P},
$$

where

$$
k_{b}=\left\{\begin{array}{c}
k_{b 1}, \text { when } b \text {-element is straight in respect to the hexagonal cell } \\
k_{b 2}, \quad \text { when } b \text {-element is slant in respect to the hexagonal cell }
\end{array}\right.
$$

is the stiffness coefficient, as described in [18], and $\mathbf{k}_{\mathbf{b}}$ and $\mathbf{m}_{\mathbf{b}}$ are the corresponding elemental stiffness and mass matrix, respectively.

Moreover, we must derive the elemental equation for the van der Waals nanosprings ( $v d w$ elements). Because this spring is only translational, we can write the elemental equation as follows:

$$
\left[\begin{array}{cc}
k_{v d w} \mathbf{I}_{20} & \mathbf{0} \\
\mathbf{0} & \mathbf{0}
\end{array}\right]\left[\begin{array}{l}
\mathbf{u} \\
\boldsymbol{\theta}
\end{array}\right]+\left[\begin{array}{ll}
\mathbf{0} & \mathbf{0} \\
\mathbf{0} & \mathbf{0}
\end{array}\right]\left[\begin{array}{l}
\ddot{\mathbf{u}} \\
\ddot{\boldsymbol{\theta}}
\end{array}\right]=\left[\begin{array}{c}
\mathbf{F} \\
\mathbf{T}
\end{array}\right] \quad \text { or } \quad \mathbf{k}_{\mathbf{v d w}} \mathbf{U}+\mathbf{m}_{\mathbf{v d w}} \ddot{\mathbf{U}}=\mathbf{P},
$$

where $k_{v d w}$ is the stiffness as derived by Equation (15) [19]. Note that the mass matrix $\mathbf{m}_{\mathbf{v d w}}$ is a null matrix because all of the inertia effects are included in the previously defined elements.

To express the stiffness matrix of the elements in the global coordinate system, a transformation matrix must be used. Let (local) nodes 1 and 2 of the axial spring correspond to nodes $i$ and $j$, respectively, of the global system. The local displacements $u_{l}$ and $u_{2}$ can be resolved into the respective components $u_{x 1}, u_{y 1}, u_{z 1}$ and $u_{x 2}, u_{y 2}, u_{z 2}$. These groups of 
components are parallel to the global $X, Y, Z$ axes, respectively. Then, the two sets of displacements are related as

$$
\mathbf{u}=\mathbf{T}_{1} \mathbf{u}_{\mathrm{xyz}}
$$

where $\mathbf{u}_{\mathbf{x y z}}$ is the vector of nodal displacements of the axial spring expressed in the global coordinate system, and the transformation matrix $\mathbf{T}$ is given by

$$
\mathbf{T}_{1}=\left[\begin{array}{cccccc}
c_{x x} & c_{y y} & c_{z z} & 0 & 0 & 0 \\
0 & 0 & 0 & c_{x x} & c_{y y} & c_{z z}
\end{array}\right]
$$

Here, $c_{x x}, c_{y y}$, and $c_{z z}$ are the direction cosines of the angles between the line $i j$ and the directions $O X, O Y$, and $O Z$, respectively. The direction cosines can be expressed with respect to the global coordinates of nodes $i$ and $j$ as

$$
\begin{aligned}
& c_{x x}=\cos (x, X)=\frac{X_{j}-X_{i}}{l_{e}} \\
& c_{y y}=\cos (x, Y)=\frac{Y_{j}-Y_{i}}{l_{e}} \\
& c_{z z}=\cos (x, Z)=\frac{Z_{j}-Z_{i}}{l_{e}}
\end{aligned}
$$

where the nanospring length is

$$
l_{e}=\sqrt{\left(X_{j}-X_{i}\right)^{2}+\left(Y_{j}-Y_{i}\right)^{2}+\left(Z_{j}-Z_{i}\right)^{2}} .
$$

The transformation matrix of the rotations for the corresponding rotational spring is similar to the one for the axial spring. The transformation matrix for a nanospring has dimension $4 \times 12$,

$$
\mathbf{T}=\left[\begin{array}{cc}
\mathbf{T}_{1} & \mathbf{0} \\
\mathbf{0} & \mathbf{T}_{1}
\end{array}\right]
$$

Hence, the elemental matrices expressed in the global coordinate system are

$$
\begin{aligned}
& \mathbf{K}_{\mathrm{a}}=\mathbf{T}^{\mathrm{T}} \mathbf{k}_{\mathrm{a}} \mathrm{T} \\
& \mathbf{M}_{\mathrm{a}}=\mathbf{T}^{\mathrm{T}} \mathbf{m}_{\mathrm{a}} \mathrm{T} \\
& \mathbf{K}_{\mathrm{b}}=\mathbf{T}^{\mathrm{T}} \mathbf{k}_{\mathrm{b}} \mathbf{T} \\
& \mathbf{K}_{\mathrm{vdw}}=\mathbf{T}^{\mathrm{T}} \mathbf{k}_{\mathbf{v d w}} \mathrm{T}
\end{aligned}
$$

The displacements and rotations as well as the loads are related by the equation

$$
\mathbf{U}=\mathbf{T D}, \mathbf{R}=\mathbf{T}^{\mathrm{T}} \mathbf{P},
$$

where $\mathbf{D}$ is the vector of displacements and rotations and $\mathbf{R}$ is the vector of loads with respect to the global coordinates. The superscript $\mathrm{T}$ in the above equations denotes matrix transposition. Finally, the elemental equations in the global system become 


$$
\begin{gathered}
K_{a} \mathbf{D}+\mathbf{M}_{\mathrm{a}} \ddot{\mathrm{D}}=\mathbf{R} \\
\mathbf{K}_{\mathrm{b}} \mathbf{D}+\mathbf{M}_{\mathbf{b}} \ddot{\mathrm{D}}=\mathbf{R} \\
\mathbf{K}_{\mathrm{vdw}} \mathbf{D}+\mathbf{M}_{\mathrm{vdw}} \ddot{\mathrm{D}}=\mathbf{R},
\end{gathered}
$$

We assemble the global stiffness $\mathbf{K}$ and global mass matrices $\mathbf{M}$ from the above elemental matrices. Considering undamped free vibration for the tubes, the equation of motion becomes:

$$
\mathbf{M} \ddot{\mathbf{X}}+\mathbf{K X}=\mathbf{0}
$$

After applying the CNT support conditions, the eigenvalue problem can be solved using common finite element procedures. The solution to the eigenvalue problem reveals the natural frequencies of vibration and the corresponding mode shapes.

\subsection{CNT with added mass modeling}

In the case that a nanoparticle is attached to a CNT mass detector, the nano-particle mass is considered to be located at a node of the CNT model and has a value $m$. Thus, for one $a_{i}$ element that includes this node, the value of the additional mass is added in Equation (16). The location of the nano-particle can be considered at the nanotube tip or at an intermediate position (Figure 5).

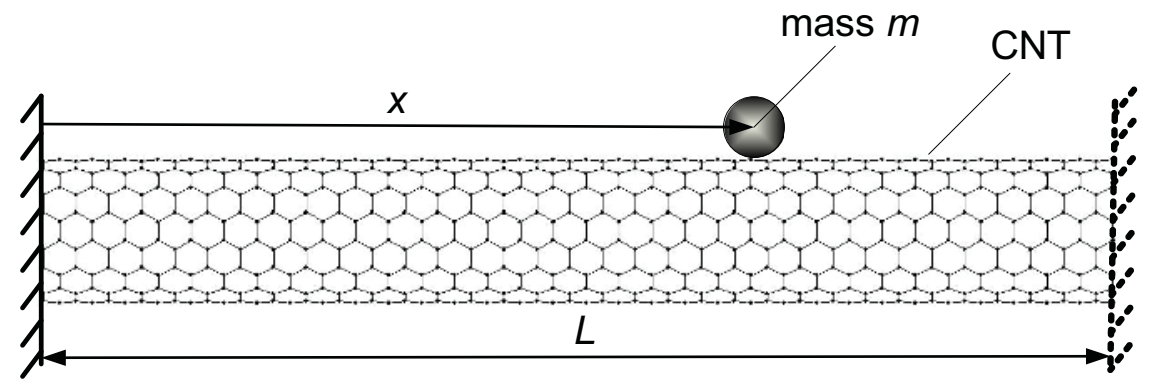

Fig. 5. CNT mass resonant sensor with an attached mass at an intermediate position.

As previously mentioned, the principle of mass detection using CNT-based detectors is based on resonant frequency shifts of a CNT due to a change in mass. The key challenge of mass detection and measuring is quantifying the changes in the resonant frequencies due to the added mass. The frequency shift is defined as

$$
\text { Frequency Shift }=\mathrm{f}_{0}-\mathrm{f},
$$

where $f_{0}$ is the frequency a CNT without an attached mass, and $f$ is the frequency of the same tube with an attached mass.

\section{Results and discussion}

\subsection{Validation of model for vibration analysis}

First, in order to validate the proposed method, we compare the results obtained from the present method with outputs from other theoretical approaches based on molecular or 
continuum mechanics, as shown in Table 1. The comparison is limited in terms of different sequence of vibration modes. This is mainly because of differences in

\begin{tabular}{|c|c|c|c|c|c|c|}
\hline \multirow{2}{*}{$\begin{array}{c}\mathrm{D}_{\mathrm{i}} \\
(\mathrm{nm})\end{array}$} & \multirow{2}{*}{$\begin{array}{c}\mathrm{D}_{\mathrm{o}} \\
(\mathrm{nm})\end{array}$} & \multirow{2}{*}{$\mathrm{L}(\mathrm{nm})$} & \multirow{2}{*}{$\begin{array}{c}\text { Support } \\
\text { Condition } \\
\text { of inner } \\
\text { CNT }\end{array}$} & \multirow{2}{*}{$\begin{array}{c}\text { Support } \\
\text { Condition } \\
\text { of outer } \\
\text { CNT }\end{array}$} & \multicolumn{2}{|c|}{$\begin{array}{c}\text { Fundamental Frequency } \\
(\mathrm{THz})\end{array}$} \\
\hline & & & & & Present & $\begin{array}{l}\text { Other } \\
\text { Studies }\end{array}$ \\
\hline 0.4 & 1.1 & 4.1 & C-C & C-C & 0.9478 & $0.9276[13]$ \\
\hline 0.4 & 1.1 & 5.5 & $\mathrm{C}-\mathrm{C}$ & $\mathrm{C}-\mathrm{C}$ & 0.6410 & $0.7355[13]$ \\
\hline 0.4 & 1.1 & 8.0 & $\mathrm{C}-\mathrm{C}$ & $\mathrm{C}-\mathrm{C}$ & 0.3551 & $0.3323[13]$ \\
\hline 0.7 & 1.4 & 14 & Free & C-C & 0.1582 & $0.1665[25]$ \\
\hline 0.7 & 1.4 & 14 & Free & $\mathrm{C}-\mathrm{F}$ & 0.0288 & 0.0270 [25] \\
\hline 0.7 & 1.4 & 14 & C-C & C-C & 0.1661 & 0.1718 [25] \\
\hline 0.7 & 1.4 & 20 & $\mathrm{C}-\mathrm{C}$ & C-C & 0.04 & $\sim 0.03[24]$ \\
\hline
\end{tabular}

Table 1. Comparison of fundamental frequencies of MWCNTs resulting from different theoretical approaches. fundamental frequencies because the other methods obtain a formulation as well as the presence of new modes that are not reported in the other methods. Very good agreement is shown between results for different support conditions.

\subsection{Modes of vibration of pure CNTs}

The solution of the eigenvalue problem reveals numerous natural frequencies and corresponding mode shapes. The lengths of studied nanotubes are appropriate for possible future miniaturized products based on nanoscale structures. Figure 6 illustrates the modes of vibration for an armchair $(12,12)$ nanotube with length $L=10.95 \mathrm{~nm}$ subjected to clamped-clamped support condition. Figure 6(a) depicts the first radial breathing mode shape in which the atoms at the half-length of the tube have the largest displacement due to the fixed-end boundary constraints. The second radial breathing mode has a vase-like shape. On the other hand, Figure 6(b) shows the first bending-like mode that simultaneously exhibits radial breathing. As a result, a triangle-like shape is observed at every cross-section of the tube. For this reason, hereafter, modes with a similar shape will be called triangular mode shapes. Furthermore, in Figure 6(c), the deformation of the nanotube has a shape in which every cross-section has a cross-like form.

For this reason, modes with this type of shape will be called as cross mode shapes of vibration. The modal analysis for the specific nanotube also reveals bending, axial, and twisting modes. The first bending mode has a half-sine shape, whereas the second has a fullsine shape, as observed in a beam. In the axial modes of vibration, the movement of the atoms is parallel to the longitudinal axis of the tube. The first axial mode is accompanied by a simultaneous movement of all atoms along the longitudinal direction. In the second one, longitudinal movement of the vectors towards the center of the tube is observed. Another type of mode is the twisting (torsional) mode, as referred to in [18]. The atoms move in the circumferential direction with a simultaneous increase in the radius of the single-walled CNT. In the first twisting mode, all atoms have the same circumferential movement and the maximum increase in the radius is observed on the half length of the tube. 


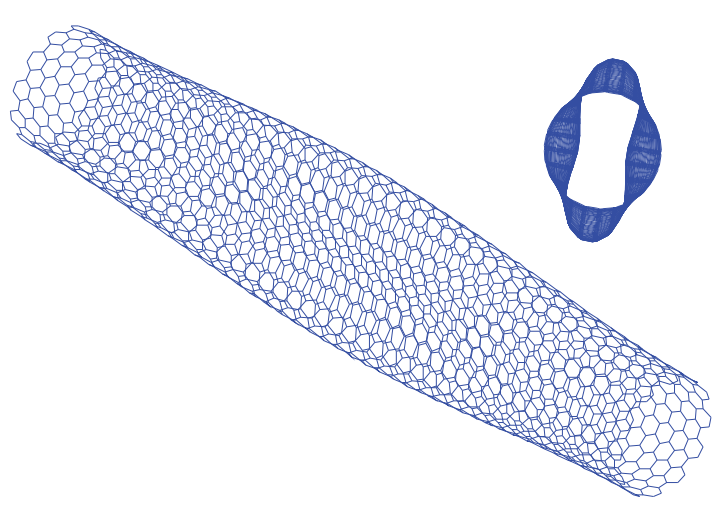

(a)

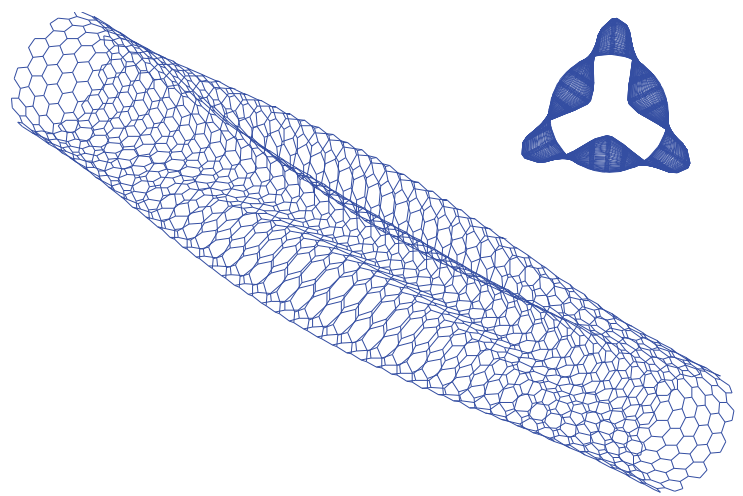

(b)

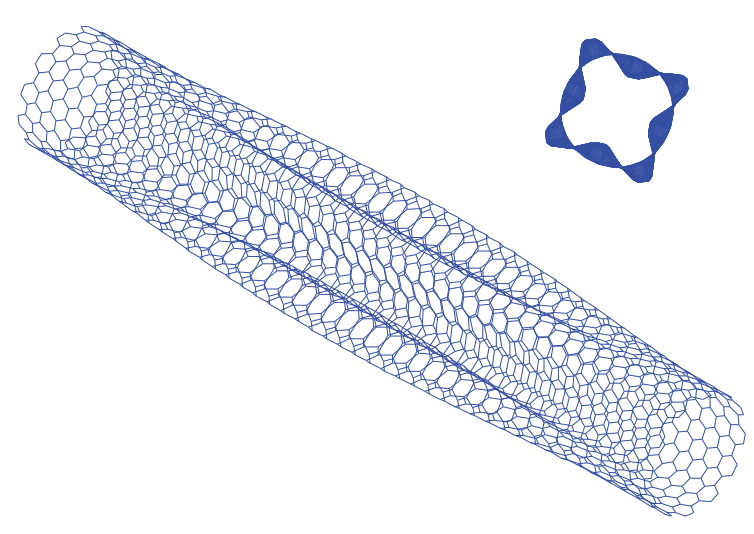

(c)

Fig. 6. Radial-like modes of vibration of clamped-clamped supported single-walled CNTs: (a) radial breathing mode, (b) triangular mode, and (c) cross mode. 


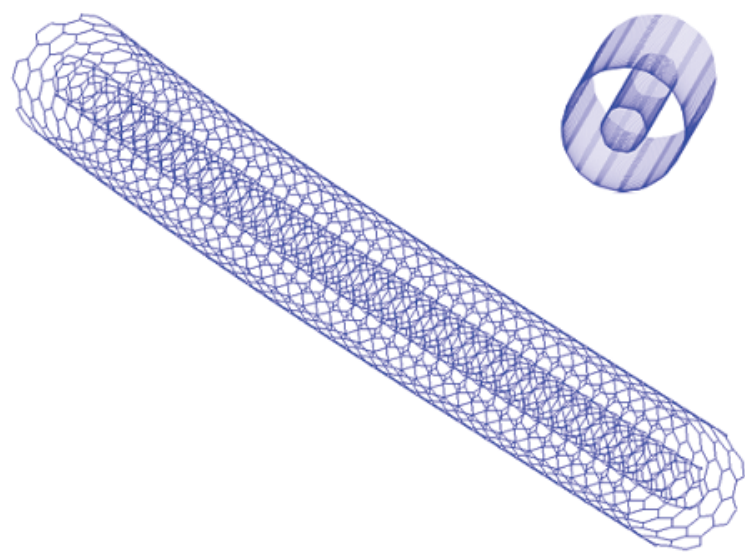

(a)

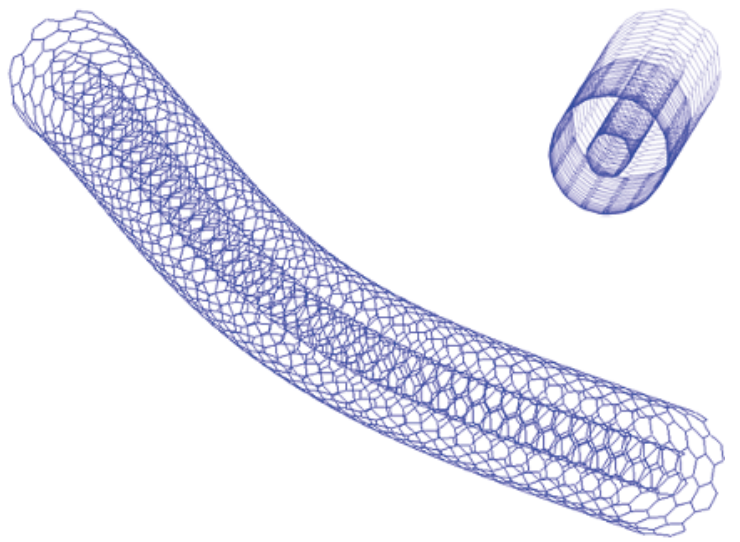

(b)

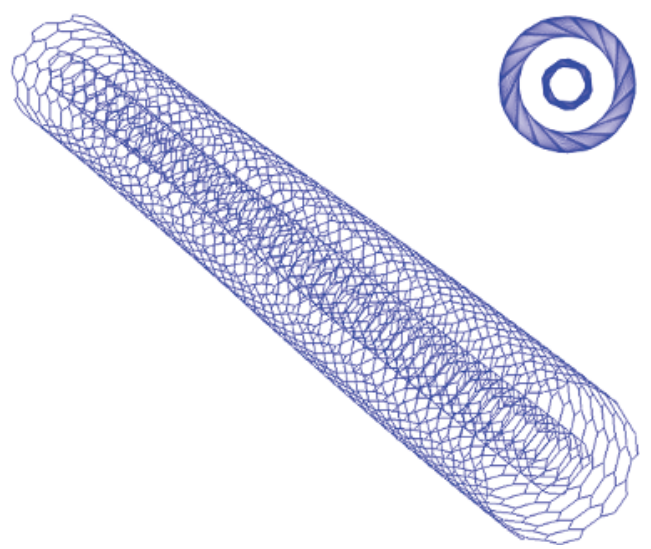

(c) 


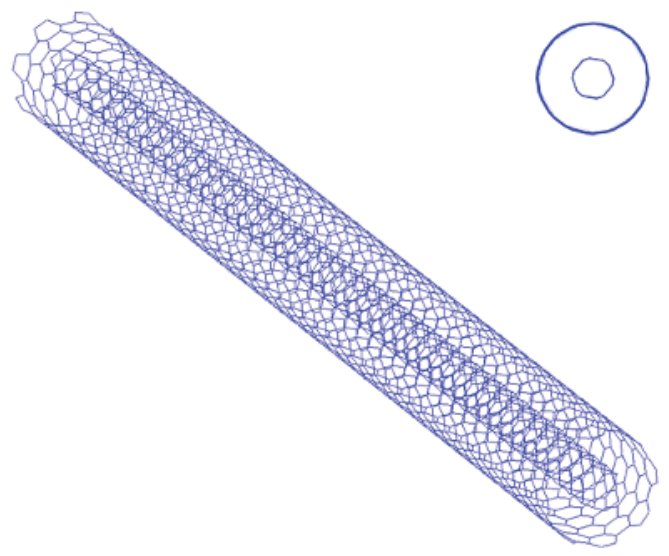

(d)

Fig. 7. Beam-like modes of vibration of clamped-free supported MWCNTs: (a) first bending, (b) second bending, (c) first twisting, and (d) first axial modes.

For the clamped-free support condition, the basic mode shapes of vibration will be described for a zigzag $(5,0)-(14,0)$ double-walled CNT with length $L=15 \mathrm{~nm}$ and using a higher aspect ratio than the previous example. Figure 7 correspondingly depicts modes that are basic for large aspect ratios. Figures $7 \mathrm{a}$ and $7 \mathrm{~b}$ illustrate the first and second bending mode shapes. These are similar to the bending modes presented in a macro-scale cantilever beam. The twisting modes (Figure 7c) exhibit a behavior similar to the clamped-clamped case, but the shapes differ because of the free end. The first axial mode (Figure $7 \mathrm{~d}$ ) is accompanied by a simultaneous movement of all atoms in the longitudinal direction and changing tube length during the vibration.

\subsection{Effect of layers on CNT vibration}

In order to investigate the influence of the number of layers on the vibration characteristics of a nanotube, CNTs of the same aspect ratio (i.e., the same length and outer diameter) were chosen for analysis with the proposed technique. Figure $8 \mathrm{a}$ depicts how the natural frequencies change for armchair tubes of length $L=17 \mathrm{~nm}$ and outer diameter $D_{o}=2.45$ nmwhen subjected to a clamped-clamped support condition. It is observed that the lower the number of layers, the lower the frequency of the first radial breathing, second radial breathing, first triangular, and first cross modes. In contrast, the higher the number of layers, the lower the frequency of the first bending, second bending, first twisting, and first axial modes. As the number of layers increases, the tube tends to behave more like a beam. Note that for tubes with only one layer (single-walled CNTs), the basic modes are the first triangular, the first cross, and the first radial breathing shapes. Figure $8 \mathrm{~b}$ illustrates similar variations for the clamped-free support condition. Here, the tube has length $L=15 \mathrm{~nm}$ and outer diameter $D_{o}=2.5 \mathrm{~nm}$. In this case, the frequencies are certainly lower, as expected with the less strict support condition. We also note that non-coaxial mode shapes are revealed, together with modes for which the inner layers exhibit different shapes from the outer ones. These are not analytically described because they are not basics modes of vibration. 


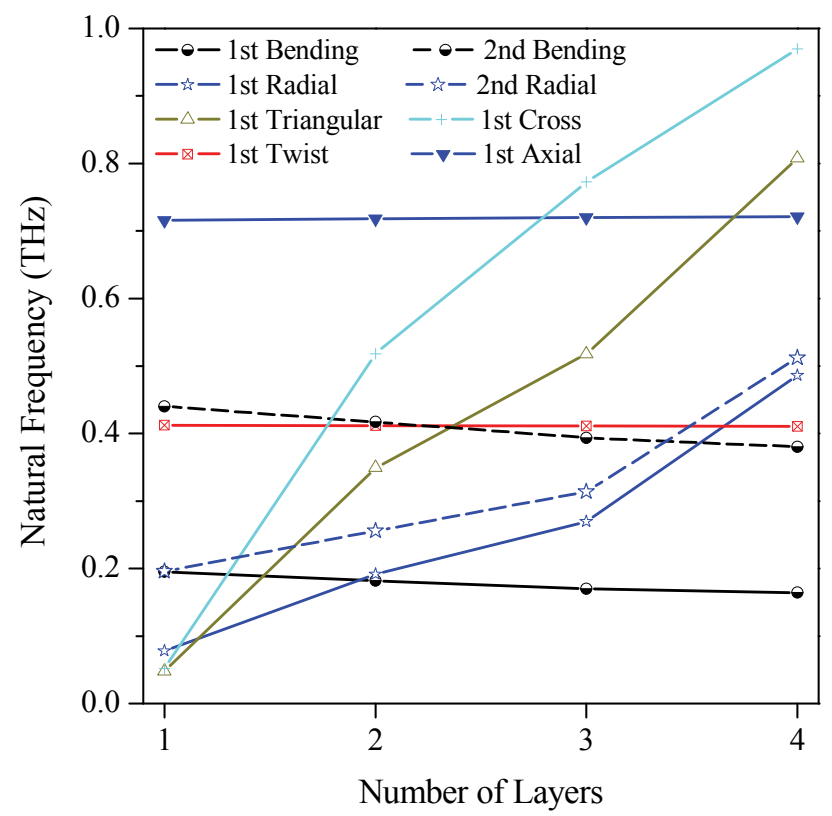

(a)

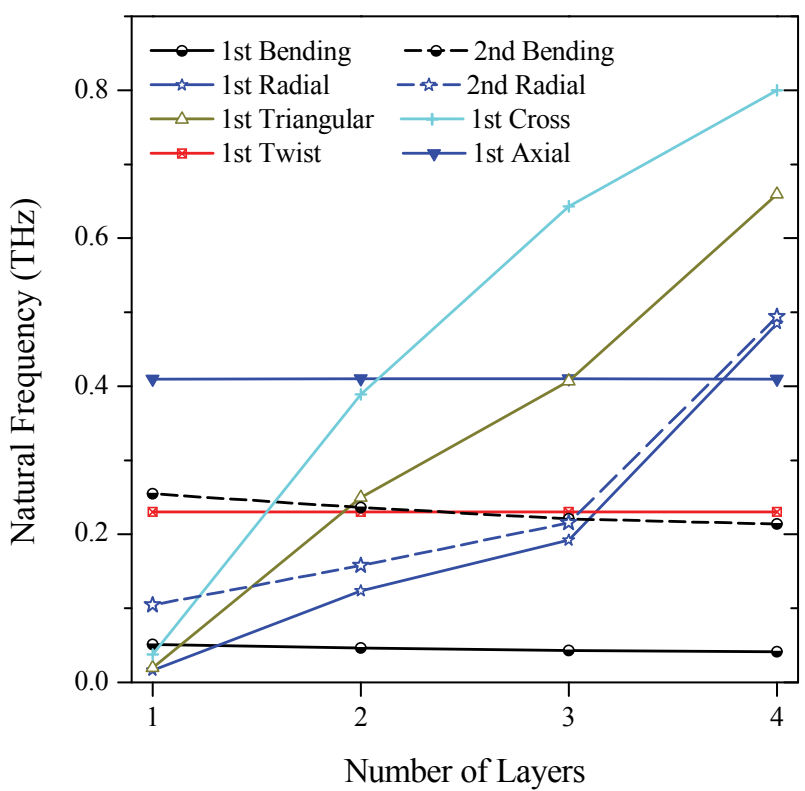

(b)

Fig. 8. Natural frequencies of (a) a clamped-clamped armchair CNT $\left(D_{o}=2.45 \mathrm{~nm}\right.$, $L=17 \mathrm{~nm})$, and (b) a clamped-free zigzag CNT $\left(D_{o}=2.5 \mathrm{~nm}, L=15 \mathrm{~nm}\right)$ vs number of layers. 


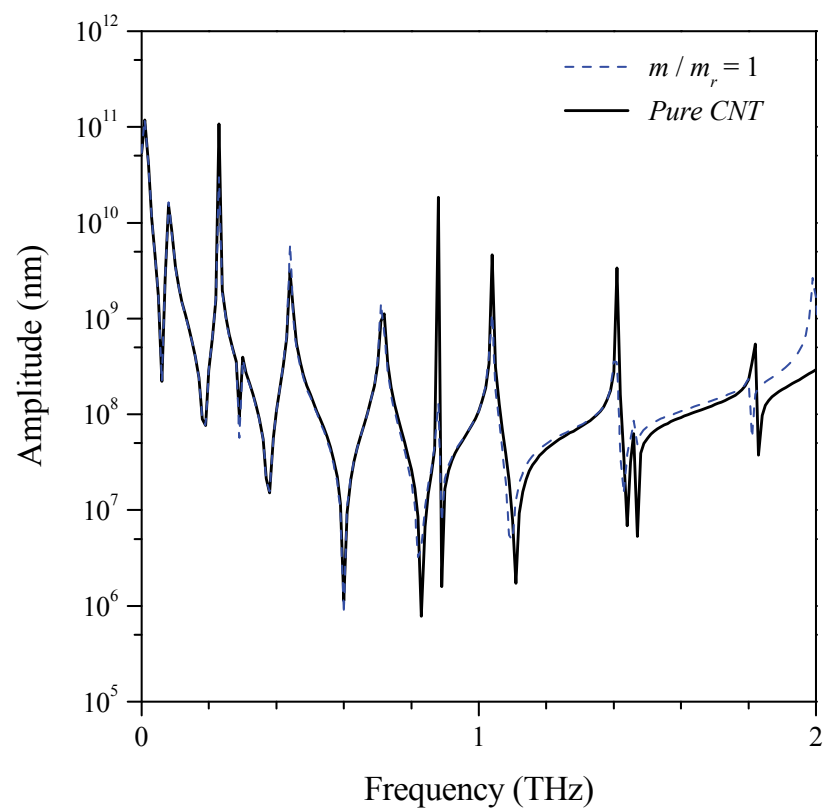

(a)

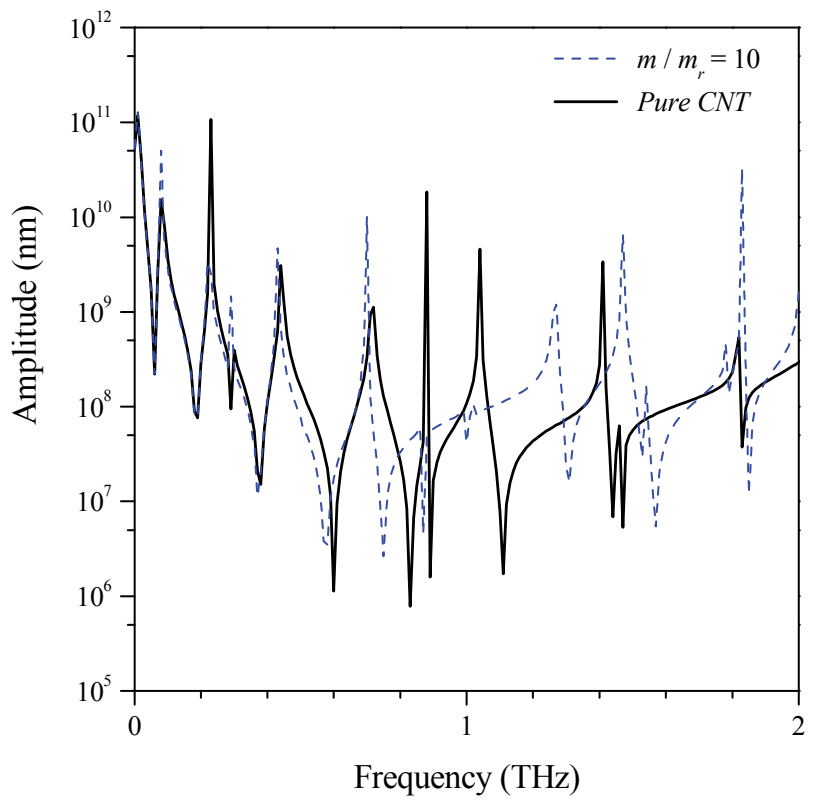

(b)

Fig. 9. Vibration spectra of clamped-free supported $(5,0) \mathrm{CNT}$ with a mass (a) $m / m_{r}=1$ and (b) $m / m_{r}=10$ attached on the CNT tip. 


\subsection{Vibration signature of CNT mass detectors}

Also, the Fast Fourier Transform (FFT) of CNT mass detector is resembled for various cases in order to be depicted the changes occurred in the spectrum due to the magnitude and location of the added mass on CNT. In Figure 9, the spectra of a simply supported zigzag $(5,0)$ with and without an added mass equal to one (Figure 9(a)) or ten carbon atoms (Figure 9(b)) attached on the tip are illustrated. The length of the CNT is $L=10.95 \mathrm{~nm}$. The continuous red line gives the response of the CNT with the added mass, while the dashed blue line gives the corresponding signature of the same, however, pure CNT.

Despite the very small value of the added mass, important changes are observed in frequencies of the fundamental and higher order modes of vibration. Here, it has to be noticed that, most of the available studies in the literature investigate mainly the fundamental frequency shift. Nevertheless, significant changes in frequencies of higher order modes are also obtained here, as in [34] also reported. The results demonstrate that the higher order frequency shifts may be very helpful in the design and function of CNT mass detectors. Similar results are obtained also for the case of clamped-clamped support condition for the same CNT, when the added mass attached on the center of the CNT length (Figure 10).

An important question, which should be answered before a practical CNT mass detector may be made, is the problem of determining where the mass lands on the nanotube. Because some parts of the tube vibrate much more than the base, a mass that lands near the fastmoving region has a much greater effect on the resonant frequency and equivalent to a much greater mass arriving near to the base. Moreover, in addition to the fundamental mode of vibration, higher-order modes in which the maximum displacement occurs at two or more positions along the nanotube are possible as have been previously seen. These higher-order vibration modes could be exploited in experiments because the change in the resonant frequency also depends on how much the nanotube moves at the absorbing point [5]. This means that measurements made with higher-order modes could potentially allow both the mass and landing position to be determined. In literature, the majority of theoretical models are focused to sense the shift of the fundamental frequency due to an added mass. However, this suffices to sense a mass addition to the CNT, but not to measure the amount of mass because the magnitude of the shift is influenced by the mass and its location on the $\mathrm{CNT}$. Hence, the sensing and measurement of the mass require investigation of the frequency shifts of the fundamental vibration mode as well as higher-order modes of vibration $[5,35]$. Based on this concept, the spectra of the CNT-mass system for different positions of the added mass on the CNT as well as different support conditions are depicted in Figure 11 and Figure 12. Here, we assume a lager mass $\left(m / m_{r}=100\right)$ than in previous results. In all cases, there are a lot of additional modes of vibration in comparison with the pure CNT case (see Figure 9). This can be explained by the asymmetry of the system existing due to the large mass value. Extremely different behaviors for different mass positions on $\mathrm{CNT}$ are observed in frequencies of the fundamental and higher order modes of vibration.

The previous mentioned results demonstrate that CNTs reveal a very different vibration signature even if a very small mass is added on its body. Moreover, they indicate that the position of the added mass noticeably influences the CNTs vibration spectra. The present methodology is capable to quantify these effects and thus can be used as a helpful computational tool for the design of CNT mass detectors. 


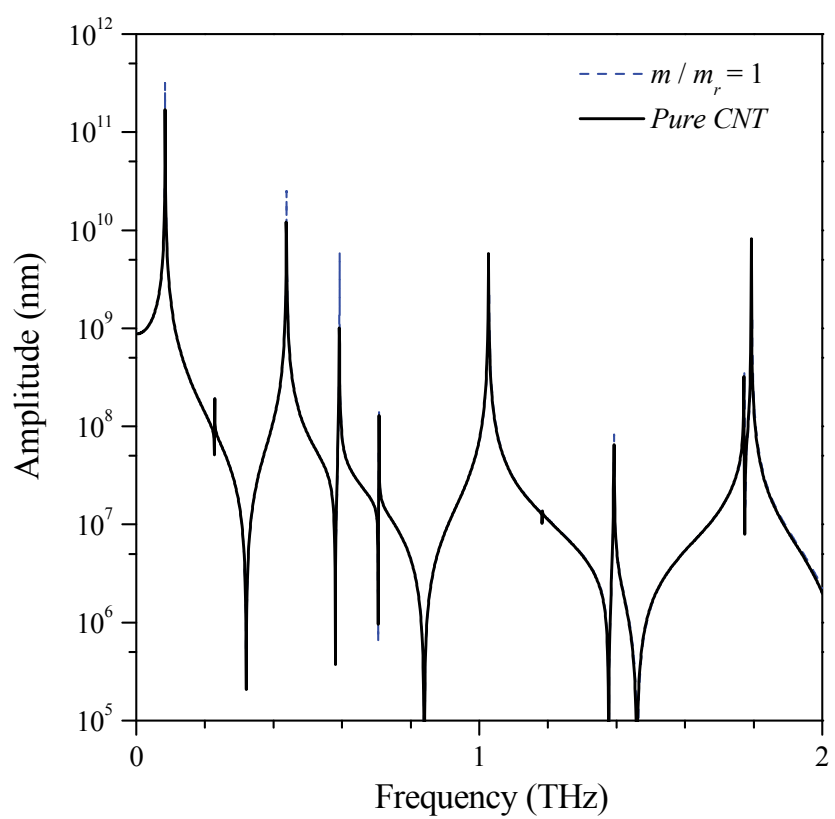

(a)

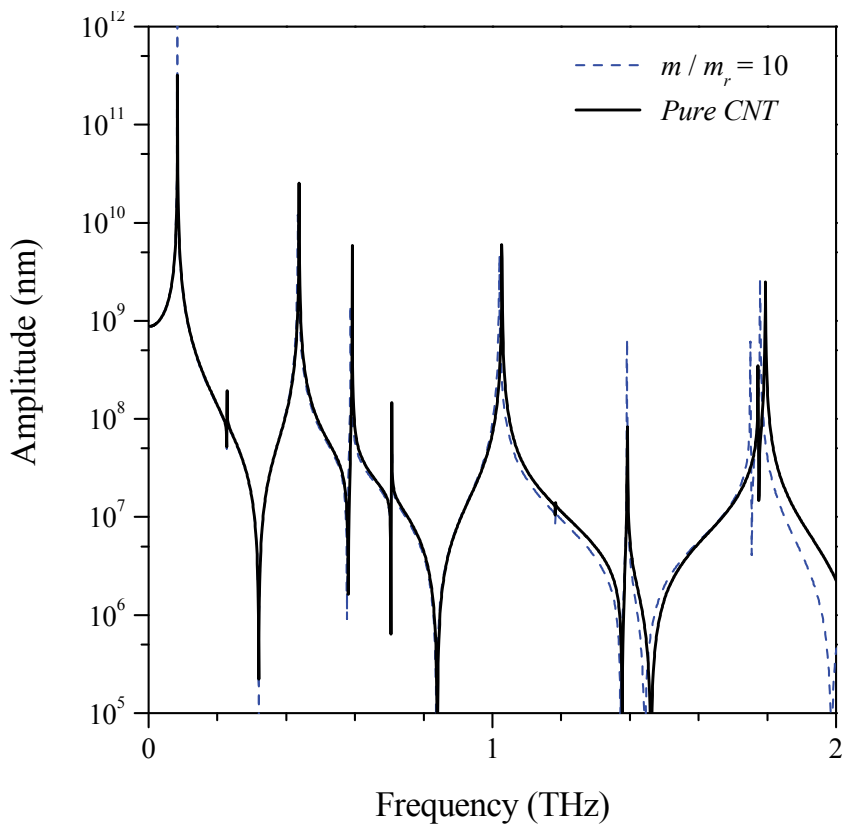

(b)

Fig. 10. Vibration spectra of clamped-clamped supported $(5,0)$ CNT with a mass (a) $m / m_{r}=1$ and (b) $m / m_{r}=10$ attached on the middle of CNT length. 


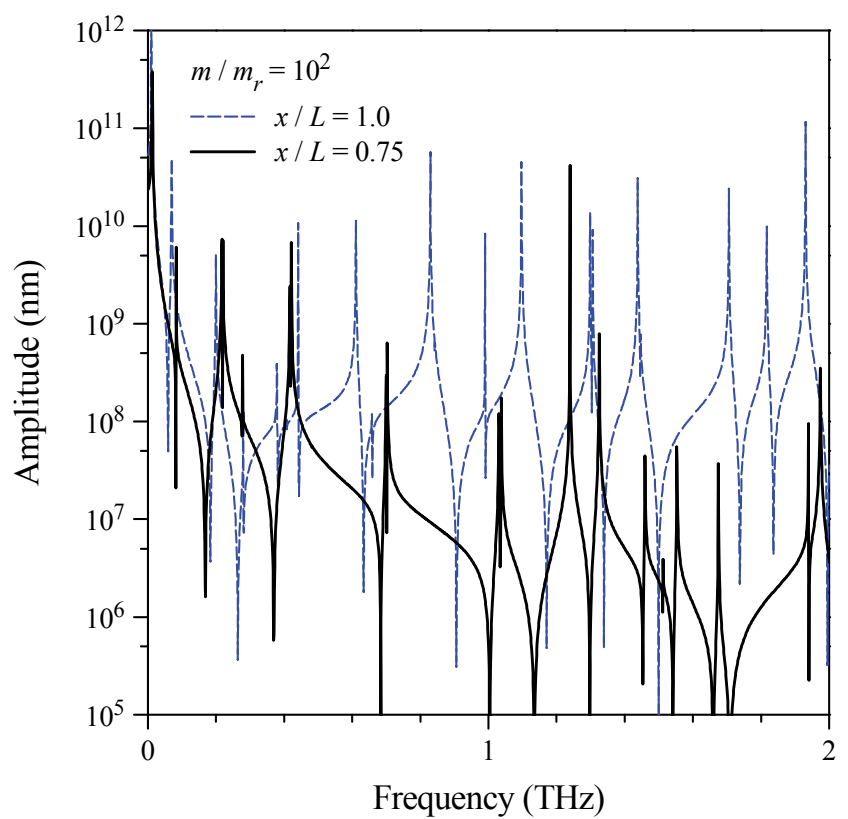

(a)

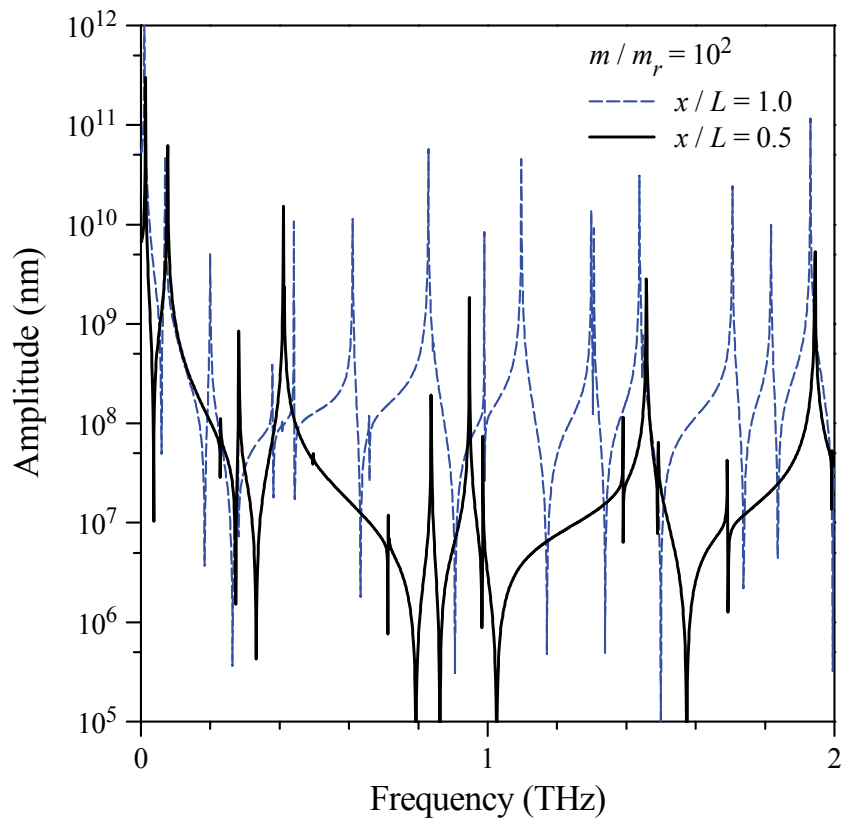

(b)

Fig. 11. Vibration spectra of clamped-free supported $(5,0) \mathrm{CNT}$ with a mass $m / m_{r}=100$ attached on the (a) $x / L=0.75$ and (b) $x / L=0.5$ position compared with CNT tip case. 


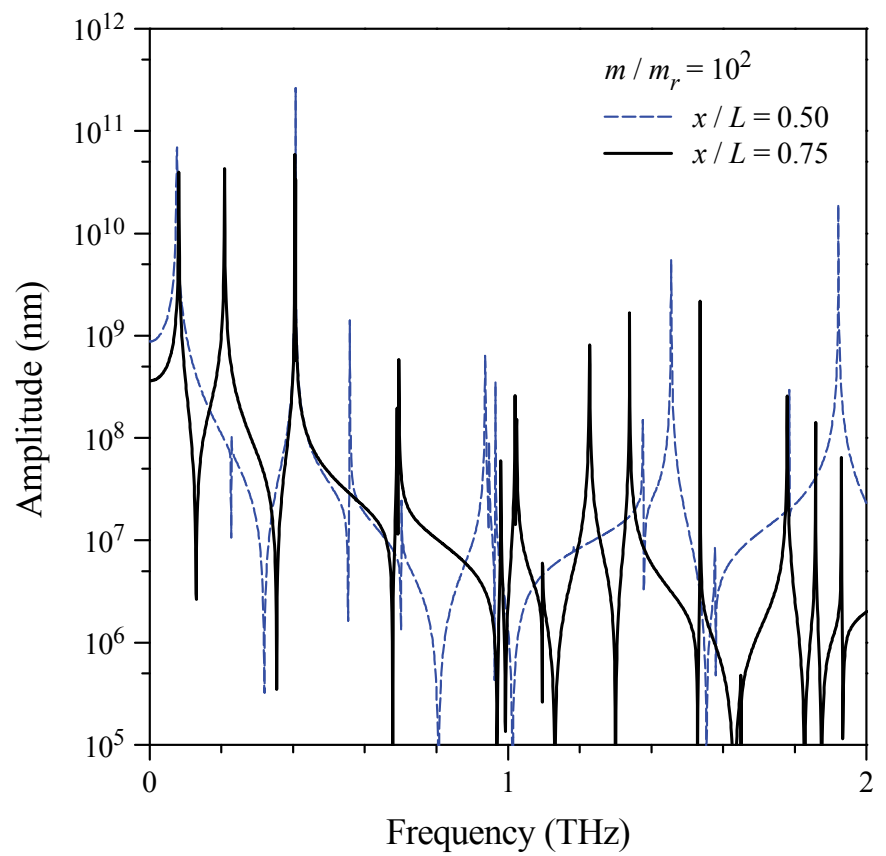

Fig. 12. Vibration spectra of clamped-clamped supported $(5,0)$ CNT with a mass $m / m_{r}=100$ attached on the $x / L=0.75$ position compared with $x / L=0.5$ case.

\begin{tabular}{|c|c|c|c|c|c|c|c|c|c|}
\hline \multirow{2}{*}{$L(\mathrm{~nm})$} & \multirow{2}{*}{$\begin{array}{c}D_{\text {in }} \\
(\mathrm{nm})\end{array}$} & \multirow{2}{*}{$\begin{array}{c}D_{\text {out }} \\
(\mathrm{nm})\end{array}$} & \multirow{2}{*}{ Layers } & \multirow{2}{*}{$\begin{array}{l}\text { Support } \\
\text { condition }\end{array}$} & \multirow{2}{*}{$\begin{array}{l}\text { Additional } \\
\text { mass } \\
\text { Position }\end{array}$} & \multicolumn{4}{|c|}{ Frequency Shift (MHz) } \\
\hline & & & & & & $\begin{array}{c}m / m r= \\
10^{0}\end{array}$ & $\begin{array}{c}m / m r= \\
10^{1}\end{array}$ & $\begin{array}{c}m / m r= \\
10^{2}\end{array}$ & $\begin{array}{c}m / m r= \\
10^{3}\end{array}$ \\
\hline \multirow{4}{*}{17} & - & \multirow{4}{*}{2.44} & 1 & \multirow{4}{*}{ C-F } & \multirow{4}{*}{ Free End } & 10 & 98 & 1208 & 7880.9 \\
\hline & 1.76 & & 2 & & & 8 & 80 & 793 & 7693 \\
\hline & 1.09 & & 3 & & & 6 & 58 & 579 & 5630 \\
\hline & 0.41 & & 4 & & & 5 & 52 & 518 & 5021 \\
\hline \multirow{4}{*}{17} & - & \multirow{4}{*}{2.44} & 1 & \multirow{4}{*}{$\mathrm{C}-\mathrm{C}$} & \multirow{4}{*}{ Middle } & 21 & 228 & 3907 & 26907 \\
\hline & 1.76 & & 2 & & & 20 & 260 & 6940 & 30420 \\
\hline & 1.09 & & 3 & & & 20 & 190 & 2800 & 19390 \\
\hline & 0.41 & & 4 & & & 20 & 170 & 2320 & 16700 \\
\hline
\end{tabular}

Table 2. Comparison of the frequency shift between single- and multi-walled CNTs with the same lengths and diameters.

\subsection{Comparison between single and multi-walled CNT mass detectors}

The main issue to examine is whether multi-walled CNTs are more or less sensitive to an added mass in terms of frequency shift than single-walled CNTs with the same geometry, 
i.e. same outer diameter and length. Based on the data included in Table 2, it can be observed that a significant difference in frequency shift exists for all of the different masses attached to the CNTs. Furthermore, a higher frequency shift was observed for CNTs with fewer layers apparently because the fractional change in mass is larger. There are cases where the frequency shift of single-walled CNTs is more than $100 \%$ higher than the corresponding shift present in a multi-walled CNT. These observations suggest that singlewalled CNTs are more suitable as mass resonant detectors than multi-walled CNTs.

\section{Conclusions}

The present chapter presented an efficient method for vibration analysis of CNTs mass detectors. The numerical method uses the CNT atomistic microstructure in order to assemble the dynamic equilibrium equation utilizing appropriate spring-mass based finite elements, which simulate the interatomic interactions and the inertia effects of CNTs. The analysis revealed the basic mode shapes of vibration and the corresponding natural frequencies of CNTs. Assuming the presence of an added mass attached on CNT, the method was utilized to resemble the FFT of CNT mass detector. The results demonstrate a significantly different vibration signature for different values and locations of the added mass in terms of frequency shift of fundamental and higher order modes. The method is capable to quantify these effects and hence could be used and facilitate the optimal design of CNT mass detectors of ultrahigh sensitivity. Finally, a parametric analysis demonstrated that single-walled carbon nanotubes are more sensitive than multi-walled CNTs and hence more suitable as mass resonant detectors.

\section{References}

[1] S. Iijima, Helical microtubes of graphite carbon, Nature 354 (1991) 56-58.

[2] R.S. Ruoff, D. Qian, W.K. Liu, Mechanical properties of carbon nanotubes: theoretical predictions and experimental measurements, C. R. Physique 4 (2003) 993-1008.

[3] M.S. Dresselhaus, G. Dresselhaus, J.C. Charlier, E. Hernandez, Electronic, thermal and mechanical properties of carbon nanotubes, Philosophical Transactions of the Royal Society A: Mathematical, Physical and Engineering Sciences 362 (2004) 2065-2098.

[4] K. Jensen, K. Kim, D.A. Zettl, An atomic-resolution nanomechanical mass sensor, Nature Nanotechnology 3 (2008) 533-537.

[5] R.G. Knobel, Weighing single atoms with a nanotube, Nature Nanotechnology 3 (2008) 525-526.

[6] R. Mateiu, A. Kuhle, R. Marie, A. Boisen, Building a multi-walled carbon nanotube-based mass sensor with the atomic force microscope, Ultramicroscopy 105 (2005) 233-237.

[7] H.-Y. Chiu, P. Hung, H.W.Ch. Postma, M. Bockrath, Atomic-Scale Mass Sensing Using Carbon Nanotube Resonators, Nano Leters 8 (2008) 4342-4346.

[8] R. Mateiu, Z.J. Davis, D.N. Madsen, K. Mølhave, P. Bøggild, A-M. Rassmusen, M. Brorson, C.J. H. Jacobsen, A Boisen, An approach to a multi-walled carbon nanotube based mass sensor, Microelectronic Engineering 73-74 (2004) 670-674.

[9] R.F. Gibson, E.O. Ayorinde, Y. Weng, Vibrations of carbon nanotubes and their composites: A review, Composites Science and Technology, 67 (2007) 1-28. 
[10] B. Liu, Y. Huand, H. Jiang, S. Qu, K.C. Hwang, The atomic-scale finite element method, Computer methods in applied mechanics and engineering, 193 (2004) 1849-1864.

[11] B. Liu, Y. Huand, H. Jiang, S. Qu, K.C. Hwang, The atomic-scale finite element method, Computer methods in applied mechanics and engineering, 193 (2004) 1849-1864.

[12] M. Mir, A. Hosseini, G.H. Majzoobi, A numerical study of vibrational properties of single-walled carbon nanotubes, Computational Materials Science, 43 (2008) 540-548.

[13] C. Li, T.-W. Chou, Vibrational behaviors of multiwalled-carbon-nanotube-based nanomechanical resonator, Applied Physics Letters, 84 (2004) 121-123.

[14] G.M. Odegard, T.S. Gates, L.M. Nicholson, K.E. Wise, Equivalent-continuum modeling of nano-structured materials, Composites Science and Technology 62 (2002) 1869-1880.

[15] C. Li, T.-W. Chou, A structural mechanics approach for the analysis of carbon nanotubes, International Journal of Solids and Structures 40 (2003) 2487-2499.

[16] K.I. Tserpes, P. Papanikos, Finite element modeling of single-walled carbon nanotubes, Composites Part B: Engineering 36 (2005) 468-477.

[17] G.I. Giannopoulos, P.A. Kakavas, , N.K. Anifantis, Evaluation of the effective mechanical properties of single walled carbon nanotubes using a spring based finite element approach, Computational Materials Science 41 (2008) 561-569.

[18] S.K. Georgantzinos, G.I. Giannopoulos, N.K. Anifantis, An efficient numerical model for vibration analysis of single-walled carbon nanotubes, Computational Mechanics 43 (2009) 731-741.

[19] S.K. Georgantzinos, N.K. Anifantis, Vibration analysis of multi-walled carbon nanotubes using a spring-mass based finite element model, Computational Materials Science 47 (2009) 168-177.

[20] T.C. Theodosiou, D.A. Saravanos, Molecular mechanics based finite element for carbon nanotube modeling, CMES - Computer Modeling in Engineering and Sciences 19(2) (2007) 121-134.

[21] C.M. Wang, V.B.C. Tan, Y.Y. Zhang, Timoshenko beam model for vibration analysis of multi-walled carbon nanotubes, Journal of Sound and Vibration, 294 (2006) 1060-1072.

[22] J. Yoon, C.Q. Ru, A. Mioduchowski, Terahertz vibration of short carbon nanotubes modeled as Timoshenko beams, Journal of Applied Mechanics, Transactions ASME, 72 (2005) 10-17.

[23] K.Y. Xu, X.N. Guo, C.Q. Ru, Vibration of a double-walled carbon nanotube aroused by nonlinear intertube van der Waals forces, Journal of Applied Physics, 99 (2006) 064303.

[24] T. Natsuki, Q.Q. Ni, M. Endo, Analysis of the vibration characteristics of double-walled carbon nanotubes, Carbon, 46 (2008) 1570-1573.

[25] K.-Y. Xu, E.C. Aifantis, Y.-H. Yan, Vibrations of double-walled carbon nanotubes with different boundary conditions between inner and outer tubes, Journal of Applied Mechanics, Transactions ASME 75 (2008) 0210131-0210139.

[26] C. Li, T.-W. Chou, Mass detection using carbon nanotube-based nanomechanical resonators, Applied Physics Letters, 84 (25) (2004) 5246-5248.

[27] D.H. Wu, W.T. Chien, C.S. Chen, H.H. Chen, Resonant frequency analysis of fixed-free single-walled carbon nanotube-based mass sensor, Sensors and Actuators A 126 (2006) 117-121.

[28] R. Chowdhury, S. Adhikari, J. Mitchell, Vibrating carbon nanotube based bio-sensors, Physica E, doi:10.1016/j.physe.2009.09.007. 
[29] J. Kołoczek, K. Young-Kyun, A. Burian, Characterization of spatial correlations in carbon nanotubes-modelling studies, Journal of Alloys and Compounds, 28 (2001) 222-225.

[30] A.K. Rappe, C.J. Casewit, K.S. Colwell, W.A. Goddard, W.M. Skiff, UFF, A full periodic table force-field for molecular mechanics and molecular dynamics simulations, Journal of American Chemical Society, 114 (1992) 10024-10035.

[31] B.R. Gelin, Molecular modeling of polymer structures and properties. Cincinnati: Hanser/Gardner Publishers, 1994.

[32] J.E. Lennard-Jones, The determination of molecular fields: from the variation of the viscosity of a gas with temperature, Proceedings of the Royal Society of London Series A 106 (1924) 441.

[33] L.A. Girifalco, R.A. Lad, Energy of cohesion, compressibility, and the potential energy functions of the graphite system, The Journal of Chemical Physics, 25 (1956) 693-697.

[34] S.K. Georgantzinos, N.K. Anifantis, Carbon nanotube-based resonant nanomechanical sensors:a computational investigation of their behavior, Physica E-Low-Dimensional Systems \& Nanostructures, 42 (2010) 1795-1801.

[35] S. Dohn, W. Svendsen, A. Boisen, O. Hansen, Mass and position determination of attached particles on cantilever based mass sensors, Review of Scientific Instruments 78 (2007) 103303. 


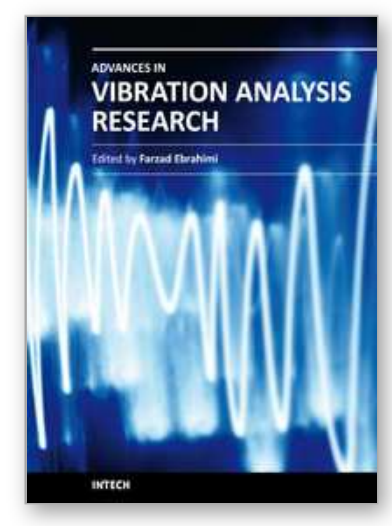

\author{
Advances in Vibration Analysis Research \\ Edited by Dr. Farzad Ebrahimi
}

ISBN 978-953-307-209-8

Hard cover, 456 pages

Publisher InTech

Published online 04, April, 2011

Published in print edition April, 2011

Vibrations are extremely important in all areas of human activities, for all sciences, technologies and industrial applications. Sometimes these Vibrations are useful but other times they are undesirable. In any case, understanding and analysis of vibrations are crucial. This book reports on the state of the art research and development findings on this very broad matter through 22 original and innovative research studies exhibiting various investigation directions. The present book is a result of contributions of experts from international scientific community working in different aspects of vibration analysis. The text is addressed not only to researchers, but also to professional engineers, students and other experts in a variety of disciplines, both academic and industrial seeking to gain a better understanding of what has been done in the field recently, and what kind of open problems are in this area.

\title{
How to reference
}

In order to correctly reference this scholarly work, feel free to copy and paste the following:

S.K. Georgantzinos and N.K. Anifantis (2011). An Atomistic-based Spring-mass Finite Element Approach for Vibration Analysis of Carbon Nanotube Mass Detectors, Advances in Vibration Analysis Research, Dr. Farzad Ebrahimi (Ed.), ISBN: 978-953-307-209-8, InTech, Available from:

http://www.intechopen.com/books/advances-in-vibration-analysis-research/an-atomistic-based-spring-massfinite-element-approach-for-vibration-analysis-of-carbon-nanotube-mas

\section{INTECH}

open science | open minds

\section{InTech Europe}

University Campus STeP Ri

Slavka Krautzeka 83/A

51000 Rijeka, Croatia

Phone: +385 (51) 770447

Fax: +385 (51) 686166

www.intechopen.com

\section{InTech China}

Unit 405, Office Block, Hotel Equatorial Shanghai

No.65, Yan An Road (West), Shanghai, 200040, China 中国上海市延安西路65号上海国际贵都大饭店办公楼 405 单元

Phone: +86-21-62489820

Fax: $+86-21-62489821$ 
(C) 2011 The Author(s). Licensee IntechOpen. This chapter is distributed under the terms of the Creative Commons Attribution-NonCommercialShareAlike-3.0 License, which permits use, distribution and reproduction for non-commercial purposes, provided the original is properly cited and derivative works building on this content are distributed under the same license. 